\title{
Near-infrared reflectance imaging of neovascular age-related macular degeneration
}

\author{
Thomas Theelen • Tos T. J. M. Berendschot • \\ Carel B. Hoyng • Camiel J. F. Boon • \\ B. Jeroen Klevering
}

Received: 9 March 2009/Revised: 9 July 2009 /Accepted: 13 July 2009 / Published online: 30 July 2009

(C) The Author(s) 2009. This article is published with open access at Springerlink.com

\begin{abstract}
Purpose To evaluate various types of neovascular agerelated macular degeneration (AMD) by near-infrared fundus reflectance (NIR) as compared to fundus fluorescein angiography (FFA) and to test NIR for assessment of leakage due to choroidal neovascularization (CNV).

Patients and methods Thirty-three patients with neovascular AMD (cases) and 20 age-matched patients with nonexudative AMD and healthy subjects (controls) were examined with a confocal scanning laser ophthalmoscope (Heidelberg Retina Angiograph 2). NIR images of neovascular AMD were qualitatively compared to the corresponding FFA and to age-matched controls. CNV membranes and exudation areas were manually segmented on FFA and NIR and analyzed quantitatively.

Results Of all cases included, five eyes had classic CNV, six had minimal classic lesions, 15 occult CNV's and seven eyes had retinal angiomatous proliferation (RAP). A dark halo on NIR was found in all cases and showed high correspondence to leakage on FFA $\left(r^{2}=0.93 ; p<0,0005\right)$. In
\end{abstract}

\footnotetext{
Meeting presentation This paper was presented in part at the 26th Congress of the Club Jules Gonin, St. Moritz, Switzerland, September 2008.

Copyright of all images included is by the authors.
}

Financial support None. No authors have any financial/conflicting interests to disclose.

T. Theelen $(\varangle) \cdot$ C. B. Hoyng • C. J. F. Boon • B. J. Klevering

Department of Ophthalmology (400),

Radboud University Nijmegen Medical Centre,

PO Box 9101, 6500 HB Nijmegen, The Netherlands

e-mail: t.theelen@ohk.umcn.nl

T. T. J. M. Berendschot

University Eye Clinique Maastricht,

Maastricht, The Netherlands classic CNV and minimal classic CNV, the classic part of the lesion on FFA revealed strong correlation to a dark core surrounded by a bright reflecting ring on NIR $\left(r^{2}=0.88\right.$; $p<0.0005)$. Occult parts on FFA of minimal classic CNV and occult CNV lesions appeared as poorly demarcated, jagged areas of increased NIR. RAP was characterized by speckled NIR located at the intraretinal neovascular complex.

Conclusions NIR imaging in neovascular AMD revealed characteristic alterations depending on the type of CNV. These changes may reflect histological differences of the lesions. Leakage caused local darkening of NIR, presumably originating from increased light-scattering and absorbance by fluid accumulation and sub-cellular structure alterations.

Keywords Age-related maculopathy Choroidal neovascularization $\cdot$ Confocal laser scanning ophthalmoscopy $\cdot$ Near-infrared reflectance . Fluorescein angiography

\section{Introduction}

Age-related macular degeneration (AMD) is the most important cause of legal blindness in elderly citizens of industrialized countries [1-3]. Progression of dry AMD to the neovascular form will lead to rapid and severe loss of central vision, and the overall prevalence of advanced agerelated macular degeneration is predicted to grow another $50 \%$ by the year 2020 [4]. Recent developments of new therapies for neovascular AMD have led to dramatic improvements in visual outcome of patients [2,5]. Modern high-quality AMD management, however, depends on accurate information about the neovascular activity. Therefore, 
detection and tracking of subretinal fluid caused by choroidal neovascularization (CNV) is crucial for evaluation and treatment of neovascular AMD.

In contrast to normal retina, the pathologic vessels of a $\mathrm{CNV}$ in neovascular AMD lack tight inter-endothelial junctions, and therefore leak $[3,6]$. As a consequence, the surrounding retina may be thickened and serously elevated, a condition that eventually leads to visual impairment. Fundus fluorescein angiography (FFA) has been the leading technique for diagnosis and follow-up of patients with neovascular MD within the last decades. FFA is used to evaluate the type of $\mathrm{CNV}$ and the presence of fluid leakage in neovascular AMD. However, classification of CNV by FFA shows significant intra- and inter-observer variability, and therefore it may not always be consistent enough for treatment evaluation [7]. In addition, intravenous dye application is necessitated for complete FFA, which makes the investigation inherently invasive.

Optical coherence tomography (OCT) has been suggested as an alternative, non-invasive imaging approach for neovascular AMD with relatively high intra- and inter-reader agreement [8]. Even though recent advances in OCT technology have facilitated three-dimensional CNV reconstruction [9], data on CNV evaluation by OCT are not directly comparable to FFA, and there may be extensive disagreement between FFA and OCT in evaluating CNV leakage [10]. This is mainly because FFA and OCT evaluate essentially different aspects of neovascular AMD. FFA assesses fluid leak directly, and therefore images the functional aspect of a $\mathrm{CNV}$, whereas indirect tissue effects like retinal thickening, elevation and cyst formation can be determined by OCT. Because such tissue alterations may also result from other pathological conditions in AMD, some OCT changes might not necessarily be the effect of active CNV leakage.

Confocal near-infrared fundus reflectance (NIR) is a noninvasive en face imaging technique capable of visualizing sub-retinal pathology [11]. NIR has been used as a rapid, non-invasive technique for imaging of neovascular AMD without injecting contrast-enhancing dyes or pupil dilation [12]. Recently, specific changes in NIR image contrast and reflectivity have been observed in AMD compared to healthy eyes, and NIR was found to be superior to standard color fundus photography in screening for neovascular AMD [13].

Several changes of NIR may be expected in neovascular AMD due to light-tissue interactions. Even though a variety of different types of neovascular AMD have been examined by NIR in the past $[12,14,15]$, a detailed description of NIR compared to FFA as a most frequently used diagnostic technique in AMD is lacking. Our present paper aims to provide a qualitative and quantitative analysis of NIR alterations and their correlation to FFA changes in different types of neovascular AMD, including assessment of lesion type and leakage.

\section{Materials and methods}

We performed a prospective, observational study of NIR and FFA in patients with newly diagnosed neovascular AMD who presented at our department between June 2007 and January 2008 and were willing to participate. Healthy individuals, as well as patients with non-exudative AMD, served as controls. Patients with prior treatment for AMD or questionable leakage were excluded. Our investigation was in accordance with the tenets of the Declaration of Helsinki and local ethics committee approval was obtained.

A second-generation confocal scanning laser ophthalmoscope (Heidelberg Retina Angiograph 2, HRA 2, Heidelberg Engineering, Heidelberg, Germany) was used to obtain both FFA and NIR images. The field of view was $30 \times 30$ degrees, and two different laser sources were applied to illuminate the ocular fundus for imaging, that is an $830 \mathrm{~nm}$ diode laser for NIR and a solid-state $488 \mathrm{~nm}$ laser with a barrier filter at $500 \mathrm{~nm}$ for FFA. All image processing and analysis was carried out using public domain software (ImageJ, v1.41d, available at http://rsb. info.nih.gov/ij). For statistical analysis SPSS 16.0 for Windows (SPSS inc., Chicago, IL, USA) was used. The Shapiro-Wilk test was used to control quantitative data for normality. Correlations between FFA and NIR measurements were visualized by scatter plots and calculated by Pearson's correlation coefficient (PCC) at the 0.01 level. To compare mean region measurements of FFA and NIR, the paired samples $t$-test was applied.

All images were acquired at the high-quality mode of HRA 2, which resulted in an original size of $1536 \times 1536$ pixels. Since no quantitative gray-value analysis was intended, images were not standardized for any confounders of image brightness like acquisition sensitivity or pupil diameter. Images were checked for eye motion artifacts and centration errors before inclusion for analysis. In the case of instable fixation, vessels may be imaged discontinuously and fundus structures may be distorted, which might lead to false measurements. Decentered images suffer from uneven illumination and may impair segmentation due to reduced local contrast. Therefore, images with the above artifacts were excluded.

For topographic inter-image comparison, images were resized by bi-cubic interpolation. For this purpose, the distance from the temporal optic disc margin to the fovea was standardized to 500 pixels. This distance has been shown to be a highly reliable anatomic marker of 3000 microns [16]. As a result, all images studied had a standardized retinal dimension of 6 microns per pixel independent of acquisition properties and refraction errors.

FFA images were classified by a highly experienced examiner $(\mathrm{CH})$ into classic, minimal classic, occult or retinal angiomatous proliferation (RAP) lesions. To reduce 
Fig. 1 FFA and NIR in controls and AMD cases. Illustration of early phase $(I)$ and late phase (II) FFAs compared to NIR (III). In normal eyes (a) NIR is homogeneous with a slight foveal dip (arrow). In dry AMD (b), no central dip was visible, but drusen caused disseminated small NIR decreases (arrows). In exudative AMD (c-f), a dark halo was present around the central lesion, corresponding to late leakage on FFA. Note the dark core surrounded by a bright corona in NIR of classic CNV (c) and the classic part of minimal classic CNV (d). The dark halo corresponding to intraretinal fluid in $\mathbf{c}$ contains a brighter, halo-like area of "normal" NIR, comparable to the surrounding fundus. This is probable due to fluid separation within the retina around the lesion. Occult lesions (e) revealed poorly demarcated speckled areas of increased NIR (arrows). The intraretinal vascular complex in RAP (f) appeared as small, focal NIR increase. The area of leakage on FFA is smaller than the dark halo on NIR (arrows). The plaque of high NIR under the leakage zone corresponds to a clinically visible choroidal nevus. Images were contrast enhanced for improved feature visibility
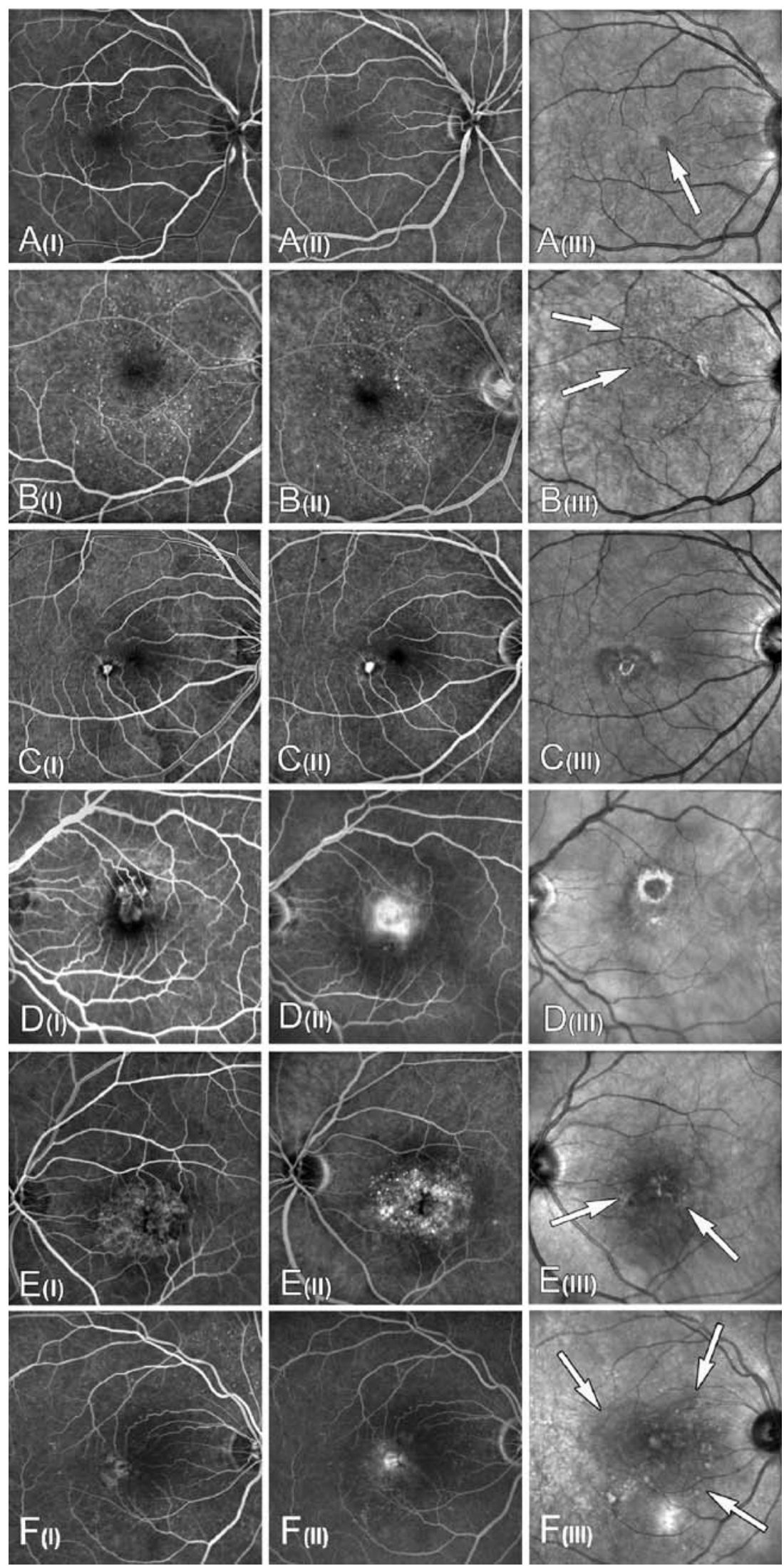
intra-observer variability [7], all FFA images were presented twice within 2 weeks in a masked, randomized fashion. Images of eyes that were not classified identically both times were rejected. Then, equally interpreted FFA's and corresponding NIR images were taken for further analysis.

As a first step, NIR images were qualitatively weighted against normal subjects and dry AMD. For this purpose, a series of age-matched eyes (ten healthy and ten dry AMD cases on FFA) were taken for comparison, and cases were presented to a single observer (TT) in a randomized and masked fashion. In this qualitative image analysis, special attention was paid to dissimilarities in NIR patterns between neovascular and dry cases.

For area measurements, segmentation was performed manually in ImageJ by polygon selection. All selections were spline fit to minimize the error of linear interpolation between individual segmentation points [17]. The borders of classic CNVs or classic parts of minimal classic CNVs were segmented on early venous phase FFAs, and fluorescein leakage was measured on FFA images taken between 7 to 8 minutes after dye injection. To identify active fluorescein leakage, early venous FFA was compared to late-phase FFA of the tested eye. In NIR, a central dark core, the surrounding bright halo and a large darkened area were segmented, if identified. To reduce bias, all NIR images were first analyzed and segmented by the masked observer (TT) without knowledge of the FFA images. Subsequently, all FFA segmentation was done 1 week later on a randomly changed image sequence. Segmented areas were measured and results were statistically analyzed.

To demonstrate the feasibility for measuring therapeutic effects with NIR, we compared a series of NIR and FFA before and after application of intravitreal injection of a vascular endothelial growth factor inhibitor (bevacizumab) in an eye with a RAP lesion. Digital subtraction maps allowed objective measurements of reflectance change on NIR parallel to diminished leakage on FFA. Measurements were based on images made with dilated pupil as well as standardized camera sensitivity, laser power and cameracornea distance.

\section{Results}

Figure 1 illustrates the NIR appearance of controls and study eyes after qualitative judgment made by one observer looking at all patients and controls in a masked and randomized order. After rejection of one eye due to intraobserver disagreement in FFA classification, and of three images because of bad NIR image quality, data analysis was based on 33 eyes (17 right and 16 left eyes) with neovascular AMD, including five eyes with classic CNV, six eyes with minimal classic CNV, 15 eyes with occult CNV and seven eyes with RAP lesions, and 20 controls (ten dry AMD and ten healthy eyes, five right and left eyes in each subgroup).

In normal eyes (Fig. 1a), NIR appeared homogenously dispersed over the posterior pole, with a slight decrease of reflectivity at the fovea. Some discrete, plaque-like increase of the NIR signal could infrequently be observed at the posterior pole. In contrast, eyes with dry AMD did not show a foveal NIR decline, and drusen appeared as disseminated, spotted NIR signal decreases (Fig. 1b). As in healthy controls, large areas of NIR reduction were missing.

All neovascular lesions (Fig. 1c-f) showed a dark halo around the center of the angiographically visible lesion. This darkened area was largely identical with the leakage zone on FFA. In all cases of classic CNV and minimal classic CNV, a lesion with a dark core bordered by a highly reflecting ring was visible and collocated to the classic CNV part on FFA (Fig. 1c,d). However, the reflectivity of the bright halo could be partially reduced in the minimal classic CNV cases. The occult parts of minimal classic $\mathrm{CNV}$ cases, as well as occult CNV lesions without detachment of the retinal pigment epithelium (RPE), appeared as jagged areas of scattered NIR increase, without definite borders to the surrounding fundus (Fig. 1d,e). In cases of RPE detachment, a sharp bordered, oval structure was observed, which showed slightly increased NIR towards its relatively ill-defined boundaries. In RAP lesions, the intraretinal vascular complex appeared to cause

Table 1 Area measurements in exudative AMD

\begin{tabular}{lllllllll}
\hline $\begin{array}{l}\text { FFA } \\
\text { classification }\end{array}$ & $\begin{array}{l}\text { CNV size [FFA] } \\
(95 \% \mathrm{CI})^{*}\end{array}$ & $\begin{array}{l}\mathrm{DC}[\mathrm{NIR}] \\
(95 \% \mathrm{CI})^{*}\end{array}$ & $P^{\dagger}$ & $\begin{array}{l}\text { DCC [NIR] } \\
(95 \% \mathrm{CI})^{*}\end{array}$ & $P^{\dagger}$ & $\begin{array}{l}\text { Leakage [FFA }] \\
(95 \% \mathrm{CI})^{*}\end{array}$ & $\begin{array}{l}\text { DH [NIR] } \\
(95 \% \mathrm{CI})^{*}\end{array}$ \\
\hline $\mathrm{CL}$ & $0.87(0.02-1.71)$ & $0.61(0.09-1.14)$ & $0.11(0.25)$ & $1.10(0.34-1.85)$ & $0.001(-0.23)$ & $4.88(3.60-6.16)$ & $5.28(4.24-6.33)$ & $0.51(-0.41)$ \\
$\mathrm{MC}$ & $0.86(0.46-1.26)$ & $0.38(0.26-0.50)$ & $0.004(0.49)$ & $0.90(0.62-1.17)$ & $0.82(-0.03)$ & $10.22(6.12-14.32)$ & $10.68(7.37-13.99)$ & $0.51(-0.46)$ \\
OC & NA & NA & NA & NA & NA & $12.78(11.06-14.51)$ & $14.00(12.12-15.88)<0.0005(-1.22)$ \\
RAP & NA & NA & NA & NA & NA & $4.25(3.43-5.06)$ & $7.75(6.34-9.17)$ & $<0.0005(-3.50)$ \\
\hline
\end{tabular}

$*=$ means of areas $\left(\mathrm{mm}^{2}\right)$ are presented together with their $95 \%$ confidence intervals; $\dagger=p$-values (and magnitude) of paired differences between NIR imaging and FFA; $C L=$ classic lesion; $\mathrm{DC}=$ dark core measured without bright corona; $\mathrm{DCC}=$ dark core measured with bright corona; $\mathrm{DH}=$ dark halo around lesion; $\mathrm{MC}=$ minimal classic lesion; $\mathrm{NA}=$ not applicable; $\mathrm{OC}=$ occult lesion; $\mathrm{RAP}=$ retinal angiomatous proliferation 
a speckled focal increase of NIR (Fig. 1f); however, this reflectance could not easily be separated from the NIR changes by a co-existing occult $\mathrm{CNV}$, if present.

Table 1 specifies detailed results on NIR and FFA measurements of the study eyes with the linear regressions shown in Fig. 2. On NIR, precise segmentation of occult lesions, RAP and RPE detachments was not possible in most cases, and therefore, quantitative analysis was restricted to leakage areas in these patients.

All quantitative results were normally distributed and analyzed by the paired-samples $t$-test. In general, NIR tended to underestimate CNV size when only the dark lesion core was compared to FFA. This was statistically significant only for minimal classic lesion parts $(p=0.004)$. On the other hand, CNV assessment by NIR resulted in significant overestimation as compared to FFA when the bright halo was included in classic lesions $(p=0.001)$. Leakage zones on FFA tended to be smaller than the darkened perilesional halo on NIR in all CNV types; however, this was only statistically significant in occult lesions and RAP $(p<0.005$ in both groups). There was strong correlation between the areas of angiographically visible classic $\mathrm{CNV}$ and the classic part of minimal classic $\mathrm{CNV}$ lesions on the one hand and the dark core lesions surrounded by bright halos evident on NIR $\left(r^{2}=0.62 ; p=\right.$ $0.007)$ on the other. The correlation appeared to be even better if the bright rings were included into the measurements $\left(r^{2}=0.88 ; p<0.0005\right)$. High correlation was observed between the large, darkened areas surrounding the neovascular lesions in NIR if weighted against the zones of late leakage on FFA $\left(r^{2}=0.93 ; p<0.0005\right)$. Digital subtraction analysis of NIR images if a RAP lesion before and after bevacizumab treatment showed reflectance elevation on NIR parallel to leakage decrease in FFA (Fig. 3).

\section{Discussion}

The ocular fundus includes a variety of absorbing, reflecting and scattering structures, which contribute to NIR and may vary considerably between individuals $[18,19]$. In contrast to visible wavelength illumination, fundus reflectance may be up to ten times higher in the near infrared, and is then largely independent of melanin content, which advances the visibility of deep fundus structures [11, 20]. In our study, NIR images of patients with neovascular AMD were analyzed and compared to lesion features on FFA.

A characteristic of neovascular membranes in AMD is intra- and subretinal fluid leak from the CNV vessels, which may be observed as fluorescein extravasation on FFA. In our present study, darkened, ill-defined areas on NIR surrounding the angiographically visible CNV showed high correlation with the late leakage zone on FFA. This
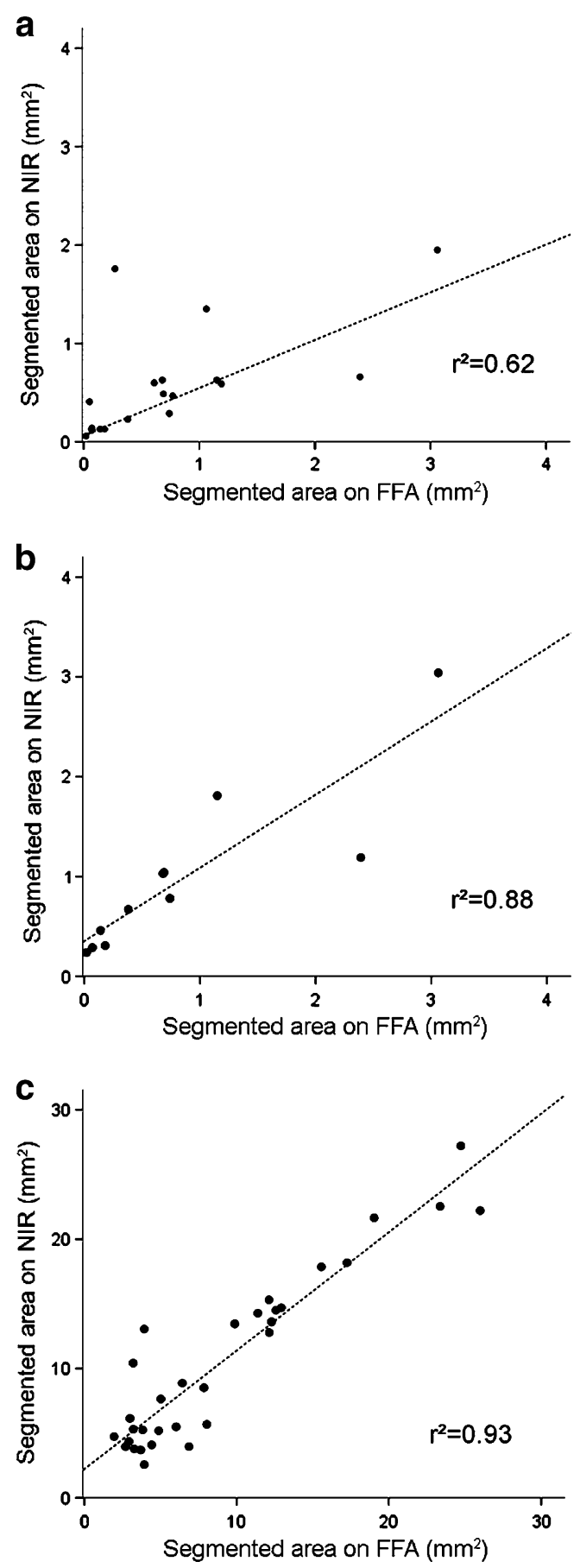

Fig. 2 Linear regression curves of $\mathrm{CNV}$ and leakage areas on FFA and NIR images. Illustration of lesion areas measured on FFA as compared to NIR. Panels $\mathbf{a}$ and $\mathbf{b}$ show data on the measures of classic neovascular membranes only without the corresponding leakage zones. There is good correlation between NIR and FFA when the size of the central dark core is compared to FFA lesion size (a). However, the correlation between FFA and NIR improves when the bright corona on NIR is added to the area measurements (b). In all cases, high correlation was observed between leakage area on FFA and the dark halo on NIR surrounding the lesion (c) 

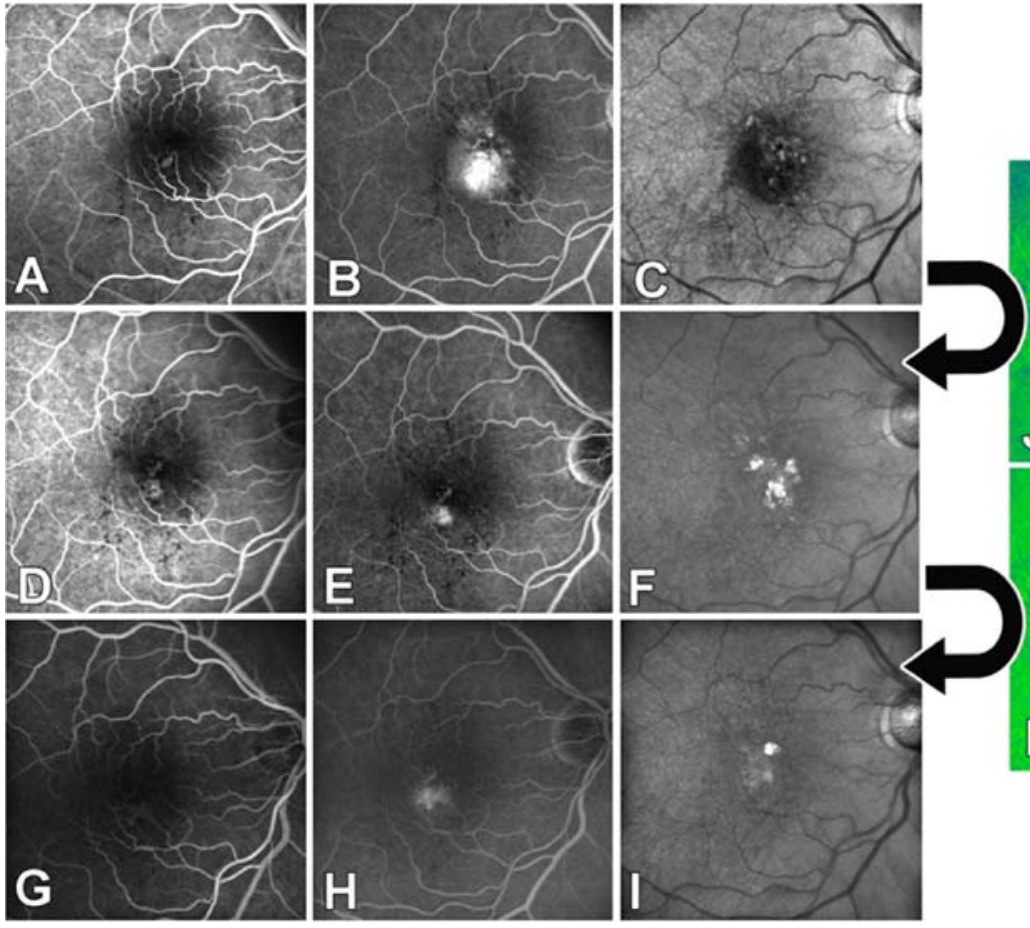
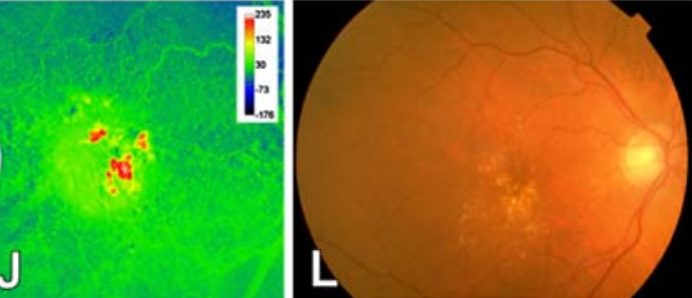

$J$
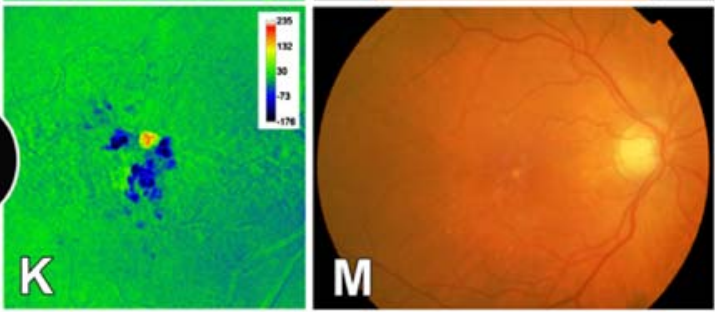

Fig. 3 Follow-up during intravitreal anti-VEGF therapy of a RAP lesion. The left image faction shows early- (left column) and latephase FFA (middle column) as well as NIR (right column) before $(\mathbf{a}-\mathbf{c})$ and after three $(\mathbf{d}-\mathbf{f})$ and six $(\mathbf{g}-\mathbf{i})$ intravitreal applications of bevacizumab. Late leakage on FFA becomes markedly reduced (b,e,h) while the initial dark halo on NIR disappears $(\mathbf{c}, \mathbf{f}, \mathbf{i})$ during treatment.

area of reduced NIR may also overlap the CNV in cases of occult lesions. Increased contents of fluid or blood will absorb near-infrared light [11]. In addition, leakage through fenestrated endothelia, as present in CNV membranes, will result in protein-rich fluid accumulation and for that reason, light-scattering will add to NIR decrease in the surrounding of an actively leaking $\mathrm{CNV}$ [14].

The halo of reduced NIR by CNV-related fluid leakage may show areas of NIR near to normal in single
Digital subtraction analysis of the standardized NIR images illustrates the increase of NIR around the RAP lesion after the first treatment series ( $\mathbf{j}$, yellow-red spots). Some foci of extreme NIR elevation, which appear as lipid exudates on funduscopy $(\mathbf{I}-\mathbf{m})$, disappear in the due course (k, blue spots). Images were contrast-enhanced for improved visualization

cases, which may be related to various reasons. First, a reactive proliferation of the underlying RPE may lead to increased NIR, and may compete NIR attenuation by fluid. Second, irregular fluid accumulation may result in unequal distribution of light absorption and scattering within the retina, thus leading to irregularly shaped NIR reduction. Summation of several optical effects may occasionally complicate the detection of fluid-related halos on NIR.

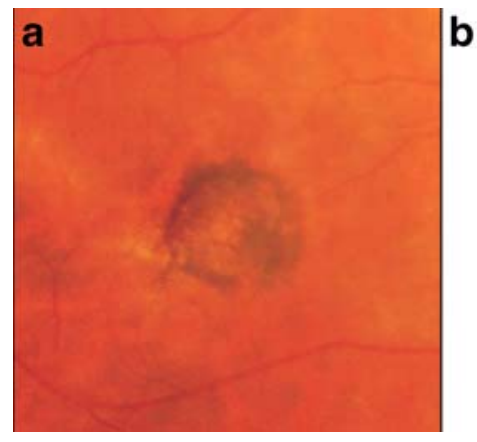

Fig. 4 Illustrated colocalization analysis of melanin and increased NIR in CNV. In a classic CNV case, regions with increased pigmentation on color fundus photograph (a) and areas of increased NIR (c) were segmented by thresholding and superimposed after image alignment (b). Total areas summed up to $0.44 \mathrm{~mm}^{2}$, where $0.32 \mathrm{~mm}^{2}$ related to melanin

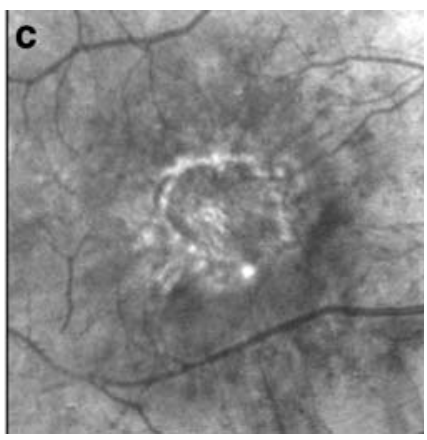

and $0.25 \mathrm{~mm}^{2}$ to NIR increase. Both areas shared $0.14 \mathrm{~mm}^{2}$, which resulted in a colocalization of $32 \%$. Images were contrast-enhanced for better performance. Segmented layers $(\mathbf{b})$ : green $=$ melanin, red $=\mathrm{NIR}$ increase, yellow $=$ shared pixels of melanin and NIR increase 
In our series, the halo of reduced NIR was significantly larger than the leakage zone on FFA in occult CNV and even more in RAP lesions. This may be caused by several reasons. Occult membranes are located within the choriocapillary layer and RPE on OCT [21]. Typically, the retina appears less thickened and flatter in occult CNV than in other types of neovascular AMD [22], which points to only mild membrane activity and leakage in most cases. FFA is capable of reporting relatively high leakage activity within several minutes; however, it will probably miss slow fluid accumulation in the surrounding area. On the other hand, NIR may detect optical changes associated with increased fluid content and therefore, affected areas of chronic leakage may appear larger than on FFA, especially in slow-leaking occult lesions. In cases with RAP, reactive, sub-cellular changes within the Müller cells may occur [23]. Such microscopic changes may increase scattering and light absorption within the inner retina prior to macroscopic thickening of the retina $[24,25]$, and thus lead to darkened areas on NIR that may appear larger than the leakage zones detected on FFA.

The total reflection of light by the ocular fundus can be divided into a directional and a non-directional part, each of which contribute to the total fundus reflectance by roughly one half [26]. Lessening of directional retinal light reflection possibly contributes to focal NIR decrease in neovascular AMD, as macular edema will confuse retinal symmetry, and therefore total retinal NIR may be even more decreased in such fundus regions. In addition, edematous changes within the retinal glial cells may add to reduced NIR by increase of intra-cellular light scatter [24].

In accordance with previous studies, all classic parts of the CNV lesions were characterized by a dark core surrounded by a bright reflecting, ring-shaped halo in NIR $[14,27,28]$. Histological analysis of excised neovascular membranes has revealed ultrastructural differences between classic and occult cases in AMD [29]. In detail, classic lesions were fibrovascular complexes located predominantly anterior to the RPE, and appeared to be surrounded by fibrin and hyperplastic, melanin-consisting RPE. The blood-rich center of such a lesion will absorb near-infrared light and therefore, will appear darker. Fibrin and melanin, on the other hand, are considered potent reflectors in the nearinfrared [30], and may therefore be represented by the bright corona of the classic CNV lesions. We compared the color fundus photographs of the classic $\mathrm{CNV}$ cases to the corresponding NIR images, and we found only poor colocalization of dark pigmented lesion components with increased NIR (Fig. 4). This gives the impression that melanin may not contribute significantly to NIR in classic CNV. Therefore, we believe that fibrin, and not melanin, should be considered the most important reflector to cause the bright $\mathrm{CNV}$ halo of NIR images.
In contrast to classic $\mathrm{CNV}$ cases, occult $\mathrm{CNVs}$ appeared as poorly demarcated areas of scattered NIR increase. In histology, occult lesions turned out to be essentially located beneath the RPE, and fibrinous material was spread diffusely over the entire surfaces of the fibrovascular membranes [29]. Assuming fibrin to be the major reflecting substance in NIR of neovascular AMD, the scattered pattern of increased NIR observed in the occult CNV cases here reflects the earlier reported ex vivo observations. In those cases where RPE detachment was present, only a slight, diffuse NIR elevation was observed at the borders of the RPE detachment (Fig. 5). A possible explanation may be that the RPE will be observed more obliquely at the borders of its detachment and therefore, any reflections from this area will sum up and increase the NIR signal.

Cases of RAP showed speckled NIR increase at the intraretinal part of the lesion. Histology of such eyes revealed fibrinous material around the neovascular complex within the retina [31]. As in other cases, this supports fibrin being a main contributor to increased NIR at or around neovascular AMD lesions. Because most RAP cases were associated with occult $\mathrm{CNV}$, a precise localization of intraretinal NIR increase was impossible, due to jagged NIR enhancement of the sub-RPE part of the membrane.

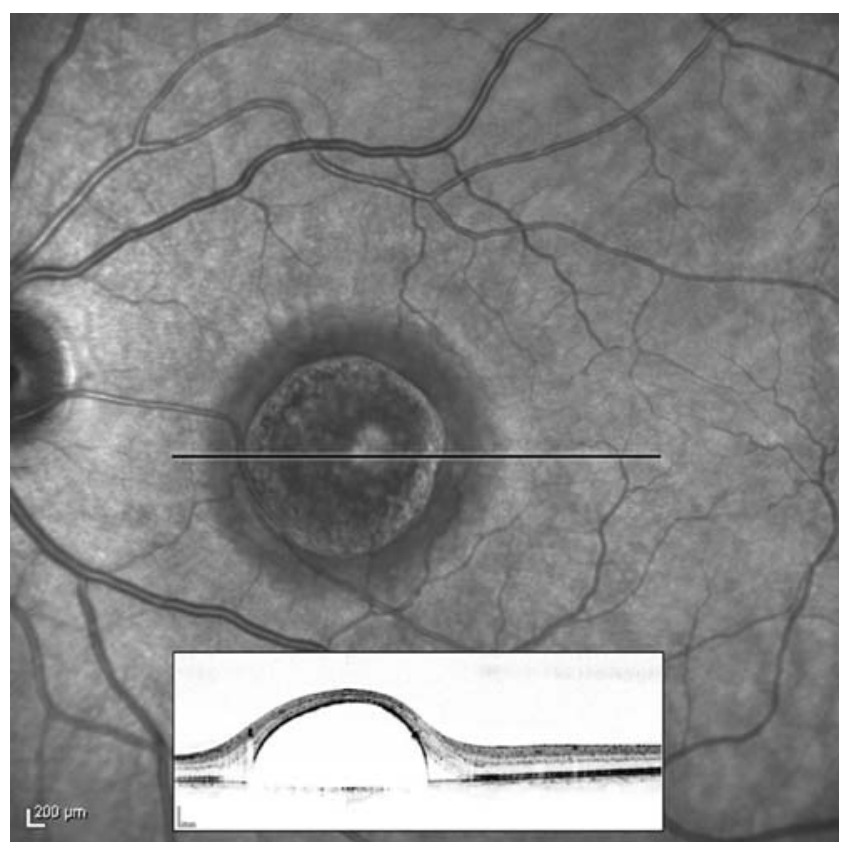

Fig. 5 Dome-shaped RPE detachment on NIR and OCT. The RPE detachment is visible as a slight, diffuse NIR elevation with a dark center, corresponding to a dome-shaped elevation of the RPE on OCT. The tip of the RPE detachment shows increased NIR due to focal light reflection. The lesion is surrounded by a dark halo, corresponding to subretinal fluid on OCT. The black line indicates the location of the OCT scan 
Reduction of leakage due to bevacizumab treatment of neovascular AMD could be demonstrated by digital subtraction analysis of NIR performed before and after treatment. In detail, previously reduced NIR collocated with the leakage zone on FFA increased, and documented the therapeutic effect in a non-invasive way. Reduced fluid content and retinal thickening led to less absorption and scattering, thus allowing more infrared light to return to the camera. In contrast to FFA, NIR does not suffer from non-leakage staining, which may be a confounder in FFA interpretation after CNV treatment [32]. Therefore, NIR appears to be a good candidate for the follow-up of neovascular AMD therapy.

Various limitations of the present study have to be addressed. First of all, the as yet incomplete understanding of $\mathrm{CNV}$ reflectivity in the near infrared limits the interpretation of our results. As in previous investigations, the interpretation of our NIR results relied on funduscopy and FFA $[15,27,30]$, but not on direct comparison with histology. Future studies have to address the topographically oriented spectral properties of NIR in neovascular $\mathrm{AMD}$, in comparison to ex vivo histology and basic optical research on reflecting substances. Secondly, the optical properties of candidate reflectors such as fibrin and melanin were hitherto not compared directly. Misinterpretation of foci of increased NIR is therefore possible. However, this does not conflict with the goal of this study, to investigate whether NIR could help to detect leakage activity, and to distinguish between several types of neovascular AMD. Nevertheless, the usefulness of NIR for neovascular AMD evaluation in daily practice remains to be determined by sensitivity and specificity assessment.

In conclusion, NIR revealed characteristic optical changes of the fundus in neovascular AMD. By this, NIR made it possible to detect leakage from active CNVs. Furthermore, distinction between different types of neovascular AMD was possible, and good spatial correlation to FFA was given in cases with classic or minimal classic $\mathrm{CNV}$. It appears likely that fibrin is the main contributor to NIR increase associated with neovascular AMD. A halo of reduced NIR around the CNV may result from light scattering and absorption within and beneath the retina due to fluid accumulation and structural tissue disorder. Masked and longitudinal studies should test NIR as an additional, non-invasive imaging technique for therapeutic studies of neovascular AMD, with the potential of refined phenotyping by imaging of sub- and intra-retinal changes.

Open Access This article is distributed under the terms of the Creative Commons Attribution Noncommercial License which permits any noncommercial use, distribution, and reproduction in any medium, provided the original author(s) and source are credited.

\section{References}

1. Klaver CC, Wolfs RC, Vingerling JR, Hofman A, de Jong PT (1998) Age-specific prevalence and causes of blindness and visual impairment in an older population: the Rotterdam Study. Arch Ophthalmol 116:653-658

2. Klein R, Klein BE, Knudtson MD, Meuer SM, Swift M, Gangnon RE (2007) Fifteen-year cumulative incidence of age-related macular degeneration: the Beaver Dam Eye Study. Ophthalmology 114:253-262

3. Jager RD, Mieler WF, Miller JW (2008) Age-related macular degeneration. N Engl J Med 358:2606-2617

4. Friedman DS, O’Colmain BJ, Munoz B, Tomany SC, McCarty C, de Jong PT, Nemesure B, Mitchell P, Kempen J (2004) Prevalence of age-related macular degeneration in the United States. Arch Ophthalmol 122:564-572

5. Schmidt-Erfurth UM, Pruente C (2007) Management of neovascular age-related macular degeneration. Prog Retin Eye Res 26:437-451

6. Campochiaro PA (2000) Retinal and choroidal neovascularization. J Cell Physiol 184:301-310

7. Holz FG, Jorzik J, Schutt F, Flach U, Unnebrink K (2003) Agreement among ophthalmologists in evaluating fluorescein angiograms in patients with neovascular age-related macular degeneration for photodynamic therapy eligibility (FLAP-study). Ophthalmology 110:400-405

8. Zhang N, Hoffmeyer GC, Young ES, Burns RE, Winter KP, Stinnett SS, Toth CA, Jaffe GJ (2007) Optical coherence tomography reader agreement in neovascular age-related macular degeneration. Am J Ophthalmol 144:37-44

9. Wojtkowski M, Srinivasan V, Fujimoto JG, Ko T, Schuman JS, Kowalczyk A, Duker JS (2005) Three-dimensional retinal imaging with high-speed ultrahigh-resolution optical coherence tomography. Ophthalmology 112:1734-1746

10. van Velthoven ME, de Smet MD, Schlingemann RO, Magnani M, Verbraak FD (2006) Added value of OCT in evaluating the presence of leakage in patients with age-related macular degeneration treated with PDT. Graefes Arch Clin Exp Ophthalmol 244:1119-1123

11. Elsner AE, Burns SA, Weiter JJ, Delori FC (1996) Infrared imaging of sub-retinal structures in the human ocular fundus. Vision Res 36:191-205

12. Kunze C, Elsner AE, Beausencourt E, Moraes L, Hartnett ME, Trempe CL (1999) Spatial extent of pigment epithelial detachments in age-related macular degeneration. Ophthalmology 106:1830-1840

13. Miura M, Elsner AE, Beausencourt E, Kunze C, Hartnett ME, Lashkari K, Trempe CL (2002) Grading of infrared confocal scanning laser tomography and video displays of digitized color slides in exudative age-related macular degeneration. Retina 22:300-308

14. Hartnett ME, Elsner AE (1996) Characteristics of exudative agerelated macular degeneration determined in vivo with confocal and indirect infrared imaging. Ophthalmology 103:58-71

15. Semoun O, Guigui B, Tick S, Coscas G, Soubrane G, Souied EH (2009) Infrared features of classic choroidal neovascularisation in exudative age-related macular degeneration. $\mathrm{Br} \mathrm{J}$ Ophthalmol 93:182-185

16. Smith RT, Chan JK, Nagasaki T, Sparrow JR, Barbazetto I (2005) A method of drusen measurement based on reconstruction of fundus background reflectance. Br J Ophthalmol 89:87-91

17. Cheney EW, Kincaid D (1994) Numerical mathematics and computing, 3rd edn., Brooks/Cole, Belmont, CA, USA

18. Delori FC, Pflibsen KP (1989) Spectral reflectance of the human ocular fundus. Appl Optics 28:1061-1077

19. van Norren D, Tiemeijer LF (1986) Spectral reflectance of the human eye. Vision Res 26:313-320 
20. van de Kraats J, Berendschot TT, van Norren D (1996) The pathways of light measured in fundus reflectometry. Vision Res 36:2229-2247

21. Coscas F, Coscas G, Souied E, Tick S, Soubrane G (2007) Optical coherence tomography identification of occult choroidal neovascularization in age-related macular degeneration. Am J Ophthalmol 144:592-599

22. Liakopoulos S, Ongchin S, Bansal A, Msutta S, Walsh AC, Updike PG, Sadda SR (2008) Quantitative optical coherence tomography findings in various subtypes of neovascular age-related macular degeneration. Invest Ophthalmol Vis Sci 49:5048-5054

23. Bringmann A, Pannicke T, Grosche J, Francke M, Wiedemann P, Skatchkov SN, Osborne NN, Reichenbach A (2006) Muller cells in the healthy and diseased retina. Prog Retin Eye Res 25:397-424

24. Franze K, Grosche J, Skatchkov SN, Schinkinger S, Foja C, Schild D, Uckermann O, Travis K, Reichenbach A, Guck J (2007) Muller cells are living optical fibers in the vertebrate retina. Proc Natl Acad Sci U S A 104:8287-8292

25. Reichenbach A, Wurm A, Pannicke T, Iandiev I, Wiedemann P, Bringmann A (2007) Muller cells as players in retinal degeneration and edema. Graefes Arch Clin Exp Ophthalmol 245:627-636

26. Berendschot TT, DeLint PJ, van Norren D (2003) Fundus reflectance-historical and present ideas. Prog Retin Eye Res 22:171-200
27. Elsner AE, Weber A, Cheney MC, Vannasdale DA, Miura M (2007) Imaging polarimetry in patients with neovascular agerelated macular degeneration. J Opt Soc Am A Opt Image Sci Vis 24:1468-1480

28. Miura M, Yamanari M, Iwasaki T, Elsner AE, Makita S, Yatagai T, Yasuno Y (2008) Imaging polarimetry in age-related macular degeneration. Invest Ophthalmol Vis Sci 49:2661-2667

29. Lafaut BA, Bartz-Schmidt KU, Vanden BC, Aisenbrey S, De Laey JJ, Heimann K (2000) Clinicopathological correlation in exudative age related macular degeneration: histological differentiation between classic and occult choroidal neovascularisation. $\mathrm{Br}$ J Ophthalmol 84:239-243

30. Weinberger AW, Lappas A, Kirschkamp T, Mazinani BA, Huth JK, Mohammadi B, Walter P (2006) Fundus near infrared fluorescence correlates with fundus near infrared reflectance. Invest Ophthalmol Vis Sci 47:3098-3108

31. Lafaut BA, Aisenbrey S, Vanden BC, Bartz-Schmidt KU (2000) Clinicopathological correlation of deep retinal vascular anomalous complex in age related macular degeneration. Br J Ophthalmol 84:1269-1274

32. Kaiser RS, Berger JW, Williams GA, Tolentino MJ, Maguire AM, Alexander J, Madjarov B, Margherio RM (2002) Variability in fluorescein angiography interpretation for photodynamic therapy in age-related macular degeneration. Retina 22:683-690 